\title{
Opioid Use Is More Common in Nonalcoholic Fatty Liver Disease Patients with Cirrhosis, Higher BMI, and Psychiatric Disease
}

\author{
Andrew M. Moon ${ }^{a}$ Stephanie E. Watkins ${ }^{b}$ Anna S. Lok ${ }^{c}$ \\ Roberto J. Firpi-Morell ${ }^{d}$ Huy Ngoc Trinhe Justin T. Kupec ${ }^{f}$ Cheryl Schoen $^{b}$ \\ Brent A. Neuschwander-Tetrig A. Sidney Barritt ${ }^{a}$ \\ aDivision of Gastroenterology and Hepatology, University of North Carolina at Chapel Hill, UNC Liver Center, Chapel

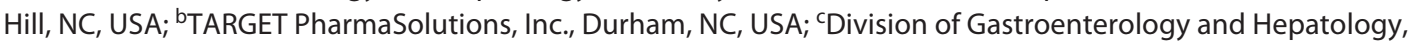 \\ University of Michigan, Ann Arbor, MI, USA; ${ }^{\mathrm{d} S e c t i o n}$ of Hepatobiliary Diseases and Transplantation, University of \\ Florida, Gainesville, FL, USA; 'San Jose Gastroenterology, San Jose, CA, USA; fDepartment of Medicine, Section of

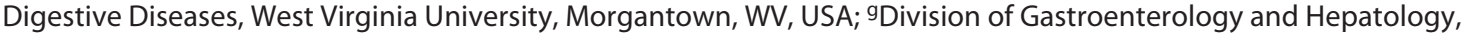 \\ Saint Louis University, St. Louis, MO, USA
}

\section{Keywords}

Fatty liver · Analgesia · Narcotics · Opiates

\begin{abstract}
Background: Opioid use is a topic of growing concern among patients with nonalcoholic fatty liver disease (NAFLD). Given safety concerns of opioids, proactively identifying subgroups of patients with an increased probability of opioid use may encourage practitioners to recommend alternative therapies for pain, thus reducing the likelihood of opioid misuse. This work assessed the prevalence and patient characteristics associated with opioid use in a real-world cohort of patients with NAFLD. Methods: TARGET-NASH, an observational study of participants at 55 academic and community sites in the United States, includes patients with NAFLD defined by pragmatic case definitions. Opioid use was defined as any documented opioid prescriptions in the year prior to enrollment. The association between patient characteristics and the odds of opioid use were modeled with stepwise multivariable logistic regression and tree ensemble methods (Classification and regression tree/Boosted Tree). Results: The cohort included 3,474 adult patients with NAFLD including $18.0 \%$ with documented opioid use. Vari-
\end{abstract}

ables associated with opioid use included presence of cirrhosis (OR 1.51, 95\% Cl 1.16-1.98), BMI $\geq 32 \mathrm{~kg} / \mathrm{m}^{2}$ (OR 1.29, 95\% Cl 1.05-1.59), depression (OR 1.87, 95\% Cl 1.50-2.33), and anxiety (OR 1.59, 95\% Cl 1.27-1.98). In the boosted tree analysis, history of back pain, depression, and fibromyalgia had the greatest relative importance in predicting opioid use. Conclusion: Prescription opioids were used in nearly 1 of 5 patients with NAFLD. Given the safety concerns of opioids in patients with NAFLD, alternative therapies including low-dose acetaminophen and nonpharmacologic treatments should be considered for these patients.

(c) 2020 S. Karger AG, Basel

\section{Introduction}

More than 50 million adults in the United States had painful chronic health conditions in 2016 [1]. Prescribing rates of opioids, commonly used in the management of patients with chronic pain, have increased in the United States since 2006 with >70,000 opioid-related overdose deaths in recent years [2]. In 2016, to improve patient safety with pain management, the Centers for Disease Control and Prevention published guidelines 
providing recommendations for alternative treatment options [3].

Nonalcoholic fatty liver disease (NAFLD) is the most common chronic liver disease worldwide [4], yet little is known regarding the prevalence of pain and associated opioid use in this population. NAFLD is commonly associated with both obesity and metabolic syndrome due to biomechanical changes which increase the mechanical load on the joints and spine, inflammatory mediators which may alter pain modulation, and poor sleep habits [5-7]. In a single center cohort of patients with NAFLD, $30 \%$ experienced pain [8]. Furthermore, model for endstage liver disease (MELD) and Child-Pugh score (CPS) have been positively associated with pain and pain-related disability [8-11].

Currently, there is a paucity of safety data, societal misconceptions, and fear of litigation associated with the treatment of chronic pain in patients with liver disease [12]. Many patients with severe liver disease receive opioid prescriptions for pain due to the increased risk of hepatic and renal toxicities associated with acetaminophen and nonsteroidal anti-inflammatory drug (NSAID) use $[8,9,13]$. Yet, use of opioids among patients with severe liver disease is associated with an increased risk of complications including prolonged hospitalization, hospital readmission, and decreased health-related quality of life [14-16].

Exercise is an alternative nonpharmacologic therapy for pain, which decreases hepatic steatosis among patients with NAFLD and improves mobility, potentially reducing the need for opioids [17-21]. In light of the safety guidelines for opioid use from the Centers for Disease Control and Prevention, there is promising evidence demonstrating that early physical therapy is associated with a reduction in future opioid use [22]. Therefore, it is possible that proactive identification of characteristics of patients at risk for opioid use may encourage health practitioners to consider alternative non-opioid therapies that may improve long-term health outcomes in these patients. This study estimated the real-world prevalence of prescription opioid use among patients with NAFLD enrolled in the TARGETNASH cohort. Additionally, this study utilized traditional modeling analyses as well as machine learning techniques to develop a patient phenotype at risk for opioid use.

\section{Methods}

\section{Cohort}

TARGET-NASH is an ongoing longitudinal cohort of adult and pediatric patients with NAFL, nonalcoholic steatohepatitis (NASH), or NAFLD-related cirrhosis managed according to lo- cal practice guidelines across academic and community gastroenterology, hepatology, and endocrinology clinics in the United States. A description of the study design has been previously published [23]. Briefly, enrolled patients consent to review of their medical records for 3 years prior to enrollment and are followed prospectively for 5 years. Clinical information from the electronic medical records, including patient narratives, laboratory results, pathology reports, and imaging data are extracted and uploaded into a secured database at 6 month intervals from the date of enrollment. Approvals from central and/or local institutional review boards are obtained prior to subject recruitment and at enrollment.

This analysis included all adult patients ( $\geq 18$ years old) enrolled in TARGET-NASH between August 1, 2016, and March 4, 2019.

\section{Case Definitions}

All patients were diagnosed by their treating physician with NAFLD based on liver biopsy and/or pragmatic case definitions. After enrollment, patients were classified as having NAFL, NASH, or NAFLD-related cirrhosis. NAFL was defined as the presence of hepatic steatosis not attributable to other causes (e.g., heavy alcohol consumption) and without evidence of biochemical and/or histological inflammation. NASH was defined as the presence of steatohepatitis on biopsy or both steatosis and elevated alanine aminotransferase levels in a patient with obesity, type 2 diabetes, dyslipidemia, or metabolic syndrome. Cirrhosis was based on clinical determination (i.e., clinical decompensation events, labs, imaging) or liver biopsy (see online suppl. Table 1; for all online suppl. material, see www.karger.com/doi/10.1159/000511074) [23].

\section{Medication Use}

Patients with documentation in the medical record of any use of the following medications codeine, buprenorphine, butorphanol, hydrocodone, dihydrocodeine, oxycodone, fentanyl, hydromorphone, methadone, morphine, oxymorphone, tramadol, pethidine, and tapentadol, within 1 year up to or at the time of enrollment were considered "opioid users." We classified these opioids into abuse potential based on scheduling categories set by the United States Drug Enforcement Administration [24]. Information on the duration of prescription opioid use was not available.

\section{Clinical Characteristics}

Medication use, medical history, and procedure and laboratory values were ascertained prior to or at enrollment. Psychiatric and medical comorbidities were determined by review of medical records for keywords within clinical notes or relevant medications. For patients with cirrhosis, CPS and MELD were calculated based on labs and clinical assessment at the time of enrollment $[25,26]$.

\section{Statistical Analysis}

The proportion of patients with documented opioid and/or non-opioid analgesic use was calculated and stratified by severity of liver disease. The distribution of opioid use by demographic characteristics, patient comorbidities, reported symptoms (abdominal pain, back pain, headache), severity of liver disease, and decompensation events was also calculated.

A $\chi^{2}$ test was used to compare the distribution of categorical variables and an ANOVA was used to compare the distribution of continuous variables among opioid users versus nonusers. 
Stepwise logistic regression was used to estimate the association between patient characteristics and the odds of receipt of opioids among all patients with NAFLD and within the strata of NAFLD cirrhosis using variables determined a priori as well as those identified via univariate analyses (significance level for entry $=0.25$; significance level to remain in the model $=0.1$ ). Collinear variables were removed, and a forward, stepwise regression model was fit. These analyses were performed using SAS version 9.4 (Cary, NC, USA).

\section{Classification and Regression Tree Analysis}

The predicted probability of opioid use at enrollment was estimated using a classification and regression tree analysis (CART) among all patients with NAFLD. A CART analysis is a data driven, nonparametric method of estimating the association between independent variables (clinical characteristics) and the predicted probability of a given outcome (opioid use). In a CART analysis, a decision tree is created using a series of dichotomous splits of patient variables that have a similar probability of opioid use [27]. At each terminal split, the algorithm predicts the outcome by classifying the response that creates the greatest difference in the prevalence of the outcome across the splitting category [28].

CART analysis has several advantages over traditional modeling techniques. First, it allows for multiple comparisons and thus can be used to assess outcomes with many possible predictor variables. Second, it does not make assumptions about the distribution or normality of the modeled outcome and it avoids the need to inform the model with transformations of predictor variables. Third, it provides modeling of the complex interactions between predictor variables, resulting in an estimated probability of use among subgroups of patients with similar characteristics, which may be more clinically applicable.

Cross validation was performed to assess the stability of the estimate. The validation data set was created using $75 \%$ of the data as the training set and $25 \%$ as the validation set with equal balance of opioid users in each group. The misclassification rate and area under the receiver operating curve were calculated for the final model.

In addition to the CART analysis, boosted trees, which applied the classification algorithm over multiple iterations of classification trees, were used to estimate the variable importance of predictors of opioid use while decreasing the prediction error of the algorithm. The following variables were included in the tree-based analysis: age, sex, race, ethnicity, BMI, recent alcohol use, Alcohol Use Disorders Identification Test (AUDIT) score, history of diabetes mellitus, depression, anxiety, osteoarthritis, rheumatologic diagnosis, dyslipidemia, gastroesophageal reflux disease, fibromyalgia, nephrolithiasis, headache, back pain, abdominal pain, ESRD, bariatric surgery, and any use of anxiolytics, antipsychotics, and antidepressants. All tree-based analyses were performed in JMP SAS (Cary, NC, USA).

\section{Results}

\section{Cohort Characteristics}

The cohort included 3,474 adult patients with NAFLD including 1,054 (30.3\%) with NAFL, 1,303 (37.5\%) with
NASH, and 1,117 (32.2\%) with NAFLD cirrhosis. At the time of enrollment, patients' mean age $( \pm$ SD) was $56.9 \pm$ 13.0 years, $58.9 \%$ were female, $74.5 \%$ white, $5.3 \%$ black, $12.3 \%$ Hispanic, and $83.9 \%$ non-Hispanic. The mean BMI was $33.3 . \pm 7.6 \mathrm{~kg} / \mathrm{m}^{2}, 50.5 \%$ of patients had diabetes, and $61.7 \%$ had dyslipidemia (Table 1 ).

\section{Characteristics of Patients by Opioid Use}

Among all patients with NAFLD, 56.1\% of patients were users of analgesics and $18.0 \%$ had documentation of opioid use within a year prior to or at enrollment. Of all opioid prescriptions, $12.2 \%$ were DEA schedule II, $1.4 \%$ were schedule III, and $7.7 \%$ were schedule IV. Opioid use was more common in patients with more advanced liver disease (12.0\% NAFL, 17.7\% NASH, 23.9\% NAFLD cirrhosis, $p<0.001)$. Opioid use was more often documented among females (20.0\%) compared to males (15.1\%) $(p<0.001)$, and opioid users had higher mean BMI (35.4 vs. $33.8 \mathrm{~kg} / \mathrm{m}^{2}, p<0.001$ ) compared to users of non-opioid analgesics. The prevalence of opioid use was highest among black race $(23.5 \%)$ compared to white $(19.9 \%)$ and other races $(6.5 \%)(p<0.001)$ (Table 1$)$.

Non-opioid analgesic use was documented in $38.1 \%$ of patients with NAFLD. Ninety percent of non-opioid analgesic users were users of NSAIDs and $12.1 \%$ were users of acetaminophen. The proportion of non-opioid analgesic use was highest among patients with NASH (42.4\%) followed by NAFLD cirrhosis $(37.5 \%)(p<0.001)(\mathrm{Ta}-$ ble 2). Patients who were users of non-opioid analgesics were 27.5 and $47.1 \%$ less likely to have a history of a mental health diagnosis or back pain, respectively, compared to opioid users (Table 1).

\section{Multivariable Model of Opioid Use in NAFLD}

In the multivariable-adjusted logistic regression model, variables associated with opioid use included NAFLD cirrhosis versus NAFL (OR 1.51, 95\% CI 1.16-1.98), BMI $\geq 32 \mathrm{~kg} / \mathrm{m}^{2}$ (OR 1.29, 95\% CI 1.05-1.59), depression (OR 1.87 , 95\% CI 1.50-2.33), anxiety (OR 1.59, 95\% CI 1.271.98 ), osteoarthritis (OR 1.42, 95\% CI 1.07-1.89), rheumatologic disease (OR 1.95, 95\% CI 1.40-2.70), gastroesophageal reflux disease (OR 1.28, 95\% CI 1.03-1.60), fibromyalgia (OR 2.17, 95\% CI 1.55-3.04), nephrolithiasis (OR 1.69, 95\% CI 1.25-2.29), back pain (OR 2.25, 95\% CI 1.76-2.87), and abdominal pain (OR 1.58, 95\% CI 1.28-1.95) (Table 3).

\section{Characteristics of Opioid Use in NAFLD Cirrhosis}

The prevalence of splenomegaly (17.2 vs. $10.1 \%, p=$ 0.003 ), hepatic encephalopathy ( 34.8 vs. $23.4 \%, p<0.001$ ), 
Table 1. Descriptive characteristics of patients with NAFLD by opioid status

\begin{tabular}{|c|c|c|c|c|c|}
\hline Summary & $\begin{array}{l}\text { Opioid use } \\
(N=624)\end{array}$ & $\begin{array}{l}\text { Other } \\
\text { Analgesic use } \\
(N=1,324)\end{array}$ & $\begin{array}{l}\text { No } \\
\text { Analgesic use } \\
(N=1,526)\end{array}$ & $\begin{array}{l}\text { All participants } \\
(N=3,474)\end{array}$ & $p$ value \\
\hline \multicolumn{6}{|l|}{ Disease severity, $n(\%)$} \\
\hline NAFLD cirrhosis & $267(42.8)$ & $419(31.6)$ & $431(28.2)$ & $1,117(32.2)$ & $<0.0001$ \\
\hline NASH & $231(37.0)$ & $553(41.8)$ & $519(34.0)$ & $1,303(37.5)$ & \\
\hline NAFL & $126(20.2)$ & $352(26.6)$ & $576(37.7)$ & $1,054(30.3)$ & \\
\hline \multicolumn{6}{|l|}{ Age at study entry, years ${ }^{1}$} \\
\hline Median $(n)$ & $58.0(624)$ & $61.0(1,324)$ & $56.0(1,525)$ & $59.0(3,473)$ & $<0.0001$ \\
\hline Mean (SD) & $57.2(11.04)$ & $59.4(12.21)$ & $54.7(13.89)$ & $56.9(12.96)$ & \\
\hline Min-max & $19.0-83.0$ & $18.0-91.0$ & $18.0-87.0$ & $18.0-91.0$ & \\
\hline \multicolumn{6}{|l|}{ Age at study entry by category, $n(\%)$} \\
\hline$<50$ & $139(22.3)$ & $233(17.6)$ & $507(33.2)$ & $879(25.3)$ & $<0.0001$ \\
\hline$\geq 50$ & $485(77.7)$ & $1,091(82.4)$ & $1,018(66.7)$ & $2,594(74.7)$ & \\
\hline Not available & 0 & 0 & $1(0.07)$ & $1(0.03$ & \\
\hline \multicolumn{6}{|l|}{ Gender, $n(\%)$} \\
\hline Female & $409(65.5)$ & $786(59.4)$ & $852(55.8)$ & $2,047(58.9)$ & $<0.0001$ \\
\hline Male & $215(34.5)$ & $538(40.6)$ & $674(44.2)$ & $1,427(41.1)$ & \\
\hline Not available & 0 & 0 & 0 & 0 & \\
\hline \multicolumn{6}{|l|}{ Race, $n(\%)$} \\
\hline White & $515(82.5)$ & $1,057(79.8)$ & $1,016(66.6)$ & $2,588(74.5)$ & $<0.0001^{20}$ \\
\hline Black or African American & $43(6.9)$ & $81(6.1)$ & $59(3.9)$ & $183(5.3)$ & \\
\hline American Indian or Alaska native & $1(0.2)$ & $6(0.5)$ & $8(0.5)$ & $15(0.4)$ & \\
\hline Asian & $14(2.2)$ & $77(5.8)$ & $335(22.0)$ & $426(12.3)$ & \\
\hline Native Hawaiian or other Pacific islander & $0(0.0)$ & $2(0.2)$ & $3(0.2)$ & $5(0.1)$ & \\
\hline Other & $22(3.5)$ & $44(3.3)$ & $53(3.5)$ & $119(3.4)$ & \\
\hline Not reported & $29(4.6)$ & $57(4.3)$ & $52(3.4)$ & $138(4.0)$ & \\
\hline Not available & 0 & 0 & 0 & 0 & \\
\hline \multicolumn{6}{|l|}{ Ethnicity, $n(\%)$} \\
\hline Hispanic or latino & $71(11.4)$ & $148(11.2)$ & $207(13.6)$ & $426(12.3)$ & 0.1749 \\
\hline Not hispanic or latino & $531(85.1)$ & $1,124(84.9)$ & $1,260(82.6)$ & $2,915(83.9)$ & \\
\hline Not reported & $14(2.2)$ & $38(2.9)$ & $45(2.9)$ & $97(2.8)$ & \\
\hline Other & $8(1.3)$ & $13(1.0)$ & $13(0.9)$ & $34(1.0)$ & \\
\hline Not available & 0 & $1(0.08)$ & $1(0.07)$ & $2(0.06)$ & \\
\hline \multicolumn{6}{|l|}{ BMI, $\mathrm{kg} / \mathrm{m}^{2}$ at enrollment } \\
\hline Median $(n)$ & $34.0(616)$ & $33.0(1,305)$ & $31.0(1,473)$ & $32.0(3,394)$ & $<0.0001$ \\
\hline Mean (SD) & $35.4(7.9)$ & $33.8(7.3)$ & $31.9(7.5)$ & $33.3(7.6)$ & \\
\hline Min-max & $17-76$ & $16-80$ & $17-103$ & $16-103$ & \\
\hline \multicolumn{6}{|l|}{ BMI, kg/m² category, $n(\%)$} \\
\hline$<32$ & $202(32.3)$ & $538(40.6)$ & $789(51.7)$ & $1,529(44.0)$ & $<0.0001$ \\
\hline$\geq 32$ & $414(66.3)$ & $767(57.9)$ & $684(44.8)$ & $1,865(53.7)$ & \\
\hline Not available & $8(1.3)$ & $19(14.4)$ & $53(3.5)$ & $80(23.0)$ & \\
\hline \multicolumn{6}{|l|}{ Alcohol usage } \\
\hline Never & $242(38.8)$ & $431(32.6)$ & $479(31.4)$ & $1,152(33.2)$ & 0.2298 \\
\hline Current & $111(17.8)$ & $326(24.6)$ & $320(21.0)$ & $757(21.8)$ & \\
\hline Former & $43(6.9)$ & $68(5.1)$ & $86(5.6)$ & $197(5.7)$ & \\
\hline Unknown & $48(7.7)$ & $98(7.4)$ & $126(8.3)$ & $272(7.8)$ & \\
\hline Not available & $180(28.8)$ & $401(30.3)$ & $515(33.7)$ & $1,096(31.5)$ & \\
\hline \multicolumn{6}{|l|}{ Smoking status } \\
\hline Never & $303(48.6)$ & $712(53.8)$ & $883(57.9)$ & $1,898(54.6)$ & $<0.0001$ \\
\hline Current & $68(10.9)$ & $92(6.9)$ & $100(6.6)$ & $260(7.5)$ & \\
\hline Former & $196(31.4)$ & $388(29.3)$ & $294(22.2)$ & $878(25.3)$ & \\
\hline Not available & $57(9.1)$ & $132(99.7)$ & $249(18.8)$ & $438(12.6)$ & \\
\hline \multicolumn{6}{|l|}{ Bariatric surgery, $n(\%)^{2}$} \\
\hline No & $595(95.4)$ & $1,287(97.2)$ & $1,502(98.4)$ & $3,384(97.4)$ & $<0.0001$ \\
\hline Yes & $29(4.6)$ & $37(2.8)$ & $24(1.6)$ & $90(2.6)$ & \\
\hline \multicolumn{6}{|l|}{ Renal failure, $n(\%)^{3}$} \\
\hline No & $611(97.9)$ & $1,311(99.0)$ & $1,514(99.2)$ & $3,436(98.9)$ & 0.0219 \\
\hline Yes & $13(2.1)$ & $13(1.0)$ & $12(0.8)$ & $38(1.1)$ & \\
\hline \multicolumn{6}{|l|}{ GERD, $n(\%)^{4}$} \\
\hline No & $180(28.8)$ & $519(39.2)$ & $884(57.9)$ & $1,583(45.6)$ & $<0.0001$ \\
\hline Yes & $444(71.2)$ & $805(60.8)$ & $642(42.1)$ & $1,891(54.4)$ & \\
\hline Dyslipidemia, $n(\%)^{5}$ & & & & & \\
\hline No & $202(32.4)$ & $348(26.3)$ & $780(51.1)$ & $1,330(38.3)$ & $<0.0001$ \\
\hline Yes & $422(67.6)$ & $976(73.7)$ & $746(48.9)$ & $2,144(61.7)$ & \\
\hline Diabetes, $n(\%)^{6}$ & & & & & \\
\hline No & $237(38.0)$ & $542(40.9)$ & $941(61.7)$ & $1,720(49.5)$ & $<0.0001$ \\
\hline Yes & $387(62.0)$ & $782(59.1)$ & $585(38.3)$ & $1,754(50.5)$ & \\
\hline
\end{tabular}


Table 1 (continued)

\begin{tabular}{|c|c|c|c|c|c|}
\hline Summary & $\begin{array}{l}\text { Opioid use } \\
(N=624)\end{array}$ & $\begin{array}{l}\text { Other } \\
\text { Analgesic use } \\
(N=1,324)\end{array}$ & $\begin{array}{l}\text { No } \\
\text { Analgesic use } \\
(N=1,526)\end{array}$ & $\begin{array}{l}\text { All participants } \\
(N=3,474)\end{array}$ & $p$ value \\
\hline \multicolumn{6}{|c|}{ Autoimmune/rheumatologic, $n(\%)^{7}$} \\
\hline No & $542(86.9)$ & $1,231(93.0)$ & $1,464(95.9)$ & $3,237(93.2)$ & \multirow[t]{2}{*}{$<0.0001$} \\
\hline Yes & $82(13.1)$ & $93(7.0)$ & $62(4.1)$ & $237(6.8)$ & \\
\hline \multicolumn{6}{|c|}{ Fibromyalgia, $n(\%)^{8}$} \\
\hline No & $530(84.9)$ & $1,246(94.1)$ & $1,497(98.1)$ & $3,273(94.2)$ & \multirow{2}{*}{$<0.0001$} \\
\hline Yes & $94(15.1)$ & $78(5.9)$ & $29(1.9)$ & $201(5.8)$ & \\
\hline \multicolumn{6}{|c|}{ Chronic fatigue, $n(\%)^{9}$} \\
\hline No & $621(99.5)$ & $1,320(99.7)$ & $1,523(99.8)$ & $3,464(99.7)$ & \multirow{2}{*}{0.2791} \\
\hline Yes & $3(0.5)$ & $4(0.3)$ & $3(0.2)$ & $10(0.3)$ & \\
\hline \multicolumn{6}{|c|}{ Chronic fatigue/fibromyalgia, $n(\%)^{10}$} \\
\hline$n$ & 624 & 1,324 & 1,526 & 3,474 & \multirow[t]{3}{*}{$<0.0001$} \\
\hline No & $528(84.6)$ & $1,243(93.9)$ & $1,495(98.0)$ & $3,266(94.0)$ & \\
\hline \multirow{2}{*}{\multicolumn{6}{|c|}{ Osteoarthritis, $n(\%)^{11}$}} \\
\hline & & & & & \\
\hline No & $509(81.6)$ & $1,148(86.7)$ & $1,462(95.8)$ & $3,119(89.8)$ & \multirow[t]{2}{*}{$<0.0001$} \\
\hline Yes & $115(18.4)$ & $176(13.3)$ & $64(4.2)$ & $355(10.2)$ & \\
\hline \multicolumn{6}{|c|}{ Nephrolithiasis, $n(\%)^{12}$} \\
\hline No & $537(86.1)$ & $1,219(92.1)$ & $1,428(93.6)$ & $3,184(91.7)$ & \multirow[t]{2}{*}{$<0.0001$} \\
\hline Yes & $87(13.9)$ & $105(7.9)$ & $98(6.4)$ & $290(8.3)$ & \\
\hline \multicolumn{6}{|c|}{ Any mental health diagnosis, $n(\%)^{13}$} \\
\hline No & $157(25.2)$ & $607(45.8)$ & $1,012(66.3)$ & $1,776(51.1)$ & \multirow[t]{2}{*}{$<0.0001$} \\
\hline \multirow{2}{*}{\multicolumn{6}{|c|}{ Any depression diagnosis, $n(\%)^{14}$}} \\
\hline & & & & & \\
\hline No & $233(37.3)$ & $762(57.6)$ & $1,146(75.1)$ & $2,141(61.6)$ & \multirow[t]{2}{*}{$<0.0001$} \\
\hline \multirow{2}{*}{\multicolumn{6}{|c|}{ Any anxiety diagnosis, $n(\%)^{15}$}} \\
\hline & & & & & \\
\hline No & $341(54.6)$ & 946 (71.) & $1,262(82.7)$ & $2,549(73.4)$ & \multirow[t]{2}{*}{$<0.0001$} \\
\hline Yes & $283(45.4)$ & $378(28.5)$ & $264(17.3)$ & $925(26.6)$ & \\
\hline \multicolumn{6}{|c|}{ Any other mental health diagnosis, $n(\%)^{16}$} \\
\hline No & $423(67.8)$ & $1,036(78.2)$ & $1,347(88.3)$ & $2,806(80.8)$ & \multirow{2}{*}{$<0.0001$} \\
\hline Yes & $201(32.2)$ & $288(21.8)$ & 179 (11.7) & $668(19.2)$ & \\
\hline \multicolumn{6}{|c|}{ Headache, $n(\%)^{17}$} \\
\hline No & $556(89.1)$ & $1,246(94.1)$ & $1,481(97.1)$ & $3,283(94.5)$ & \multirow[t]{2}{*}{$<0.0001$} \\
\hline Yes & $68(10.9)$ & $78(5.9)$ & $45(2.9)$ & $191(5.5)$ & \\
\hline \multicolumn{6}{|c|}{ Back pain, $n(\%)^{18}$} \\
\hline No & $453(72.6)$ & $1,132(85.5)$ & $1,443(94.6)$ & $3,028(87.2)$ & $<0.0001$ \\
\hline Yes & $171(27.4)$ & $192(14.5)$ & $83(5.4)$ & $446(12.8)$ & \\
\hline Abdominal & & & & & \\
\hline No & $391(62.7)$ & 995 (75.2) & $1,258(82.4)$ & $2,644(76.1)$ & $<0.0001$ \\
\hline Yes & $233(37.3)$ & $329(24.8)$ & 268 (17.6) & $830(23.9)$ & \\
\hline Acetomino & & & & & \\
\hline No & $362(58.0)$ & $1,164(87.9)$ & $1,526(100.0)$ & $3,052(87.9)$ & \\
\hline Yes & $262(42.0)$ & $160(12.1)$ & $0(0.0)$ & $422(12.1)$ & \\
\hline NSAID use & & & & & \\
\hline No & $263(42.1)$ & $229(17.3)$ & $1,526(100.0)$ & $2,018(58.1)$ & \\
\hline Yes & 361 (57.9) & $1,095(82.7)$ & $0(0.0)$ & 1,456 (41.9) & \\
\hline
\end{tabular}

Opioid use reported within 1 year prior to or at enrollment. Other medication use, medical history, procedures and laboratory values reported on or prior to enrollment. NASH, nonalcoholic steatohepatitis; NAFLD, nonalcoholic fatty liver disease; NSAID, nonsteroidal anti-inflammatory drug; GERD, gastroesophageal reflux disease. ${ }^{1}$ Age calculated based on year of consent minus birth year. ${ }^{2}$ From procedures coded as "Gastric banding" or "Gastric bypass." ${ }^{3}$ Medical history coded as "Renal failure." ${ }^{4}$ Medical history coded as "Gastroesophageal reflux disease" or any use of a proton pump inhibitor. ${ }^{5}$ Medical history coded terms indicative of dyslipidemia or any use of medications indicative of dyslipidemia. ${ }^{6}$ Medical history coded to diabetes (excluding prediabetes or insulin-resistant diabetes), medications used to treat diabetes or HbAlc $>6.5 .{ }^{7}$ Medical history coded to terms indicative of autoimmune/rheumatologic conditions. ${ }^{8}$ Medical history coded to "Fibromyalgia." ${ }^{9}$ Medical history coded to "Chronic fatigue syndrome." ${ }^{10}$ Medical history coded to "Chronic fatigue syndrome" or "Fibromyalgia." 11 Medical history coded to "Osteoarthritis." 12 Medical history coded to "Nephrolithiasis." ${ }^{13}$ Medical history coded to "Psychiatric disorders" or medications used to treat mental health disorders. ${ }^{14}$ Medical history indicative of depression or medications used to treat depression. ${ }^{15}$ Medical history indicative of anxiety or medications used to treat anxiety. ${ }^{16}$ Medical history coded to "Psychiatric disorders" that are not indicative of depression or anxiety, or medications used to treat schizophrenia. ${ }^{17}$ Medical history coded terms indicative of headache. ${ }^{18}$ Medical history coded terms indicative of back pain. ${ }^{19}$ Medical history coded terms indicative of abdominal pain. ${ }^{20}$ Race grouped as white, black or African American, or other. 
Table 2. Frequency of analgesic use overall and by NAFLD category

\begin{tabular}{|c|c|c|c|c|c|}
\hline & $\begin{array}{l}\text { Overall } \\
(N=3,474) \\
N(\%)\end{array}$ & $\begin{array}{l}\text { NAFLD cirrhosis } \\
(N=1,117) \\
N(\%)\end{array}$ & $\begin{array}{l}\text { NASH } \\
(N=1,303) \\
N(\%)\end{array}$ & $\begin{array}{l}\text { NAFL } \\
(N=1,054) \\
N(\%)\end{array}$ & $p$ values \\
\hline Opioid use ${ }^{1}$ & $624(18.0)$ & 267 (23.9) & $231(17.7)$ & $126(12.0)$ & $<0.001$ \\
\hline Non-opioid analgesic use ${ }^{2}$ & $1,324(38.1)$ & $419(37.5)$ & $553(42.4)$ & $352(33.4)$ & $<0.001$ \\
\hline No analgesic use & $1,526(43.9)$ & $431(38.6)$ & $519(39.8)$ & $576(54.6)$ & $<0.001$ \\
\hline
\end{tabular}

NASH, nonalcoholic steatohepatitis; NAFLD, nonalcoholic fatty liver disease; GERD, gastroesophageal reflux disease. ${ }^{1}$ Opioid use within 1 year prior to enrollment or at the time of enrollment. ${ }^{2}$ Excludes patients with prior opioid use.

Table 3. Odds of opioid use among patient with NAFLD by patient characteristics*

\begin{tabular}{lll}
\hline & OR & $95 \% \mathrm{CI}$ \\
\hline NAFLD severity & & \\
$\quad$ NAFL & Reference & Reference \\
NASH & 1.15 & $0.89-1.50$ \\
$\quad$ NAFLD cirrhosis & $\mathbf{1 . 5 1}$ & $\mathbf{1 . 1 6}-\mathbf{1 . 9 8}$ \\
Age $\geq 59$ years (vs. $<59)$ & 0.86 & $0.70-1.06$ \\
BMI $\geq 32$ kg/m ${ }^{2}$ (vs. $\left.<32\right)$ & $\mathbf{1 . 2 9}$ & $\mathbf{1 . 0 5}-\mathbf{1 . 5 9}$ \\
Diabetes mellitus & 1.21 & $0.98-1.49$ \\
Depression & $\mathbf{1 . 8 7}$ & $\mathbf{1 . 5 0}-\mathbf{2 . 3 3}$ \\
Anxiety & $\mathbf{1 . 5 9}$ & $\mathbf{1 . 2 7 - 1 . 9 8}$ \\
Osteoarthritis & $\mathbf{1 . 4 2}$ & $\mathbf{1 . 0 7 - 1 . 8 9}$ \\
Rheumatologic diagnosis & $\mathbf{1 . 9 5}$ & $\mathbf{1 . 4 0 - 2 . 7 0}$ \\
Fibromyalgia & $\mathbf{2 . 1 7}$ & $\mathbf{1 . 5 5}-\mathbf{3 . 0 4}$ \\
GERD & $\mathbf{1 . 2 8}$ & $\mathbf{1 . 0 3}-\mathbf{1 . 6 0}$ \\
Nephrolithiasis & $\mathbf{1 . 6 9}$ & $\mathbf{1 . 2 5}-\mathbf{2 . 2 9}$ \\
Back pain & $\mathbf{2 . 2 5}$ & $\mathbf{1 . 7 6 - 2 . 8 7}$ \\
Abdominal pain & $\mathbf{1 . 5 8}$ & $\mathbf{1 . 2 8}-\mathbf{1 . 9 5}$ \\
\hline
\end{tabular}

Variables included in the stepwise model: disease severity, age(years), sex, race (white vs. non-white), BMI, type 2 diabetes, osteoarthritis, depression, autoimmune/rheumatologic disease, hyperlipidemia, GERD, fibromyalgia, nephrolithiasis, anxiety, headache, back pain and abdominal pain: significance level to remain in the model $=0.1{ }^{*}$ Patient characteristics were ascertained from the medical record at or prior to enrollment. NASH, nonalcoholic steatohepatitis; NAFLD, nonalcoholic fatty liver disease; GERD

and ascites ( 43.1 vs. $34.4 \%, p=0.010$ ) was significantly higher among opioid users compared to patients without documentation of opioid use. The prevalence of hepatocellular carcinoma was not significantly different among patients with cirrhosis who had documentation of opioid use compared to nonusers ( 3.4 vs. $2.0 \%, p=0.214$ ).
Among opioid users and nonusers with NAFLD cirrhosis, there was no significant difference in mean MELD score (10.3 vs. $10.1, p=0.501)$ or CPS ( 8.0 vs. $7.7, p=$ 0.195) (Table 4).

\section{Multivariable Model of Opioid Use in NAFLD Cirrhosis}

In the multivariate analysis of opioid use among patients with NAFLD cirrhosis, age, sex, BMI, osteoarthritis, rheumatologic diagnosis, depression, MELD, ascites, HCC, and hepatic encephalopathy were considered in our initial model (Table 5). In our final model, age $>62$ years (OR 0.70, 95\% CI 0.51-0.96), depression (OR 2.29, 95\% CI 1.66-3.18), rheumatologic disease (OR 2.53, 95\% CI 1.48-4.30), and osteoarthritis (OR 2.30, 95\% CI 1.533.46) were associated with opioid use (Table 5).

\section{Classification and Boosted Tree Analysis}

In the boosted tree analysis, a history of fibromyalgia, depression, or back pain in the medical record had the greatest variable importance for predicting opioid use over 100 trees at a sampling rate of 0.75 . The area under the receiver operating curve was 0.76 for the learning sample and 0.73 for the validation sample. The misclassification rate was 0.18 for both the training and the validation sample (Fig. 1).

Forty six percent of patients with NAFLD who had a history of depression and back pain were opioid users. The probability of opioid use among patients with a combined history of depression and fibromyalgia without back pain was $44 \%$. Among patients without depression, the probability of opioid use was approximately $20 \%$ for patients with anxiety, abdominal pain, or back pain. The misclassification rate in the training sample and validation sample was 0.18 (Table 6). 
Table 4. Characteristics of opioid users versus nonusers with NAFLD cirrhosis*

\begin{tabular}{lcll}
\hline & $\begin{array}{l}\text { Opioid use } \\
(n=267) \\
N(\%)\end{array}$ & $\begin{array}{l}\text { No opioid use } \\
(n=850)\end{array}$ & $p$ value \\
& $N(\%)$ & $N(\%)$ \\
\hline MELD (mean) (SD) & $10.3(4.5)$ & $10.1(4.0)$ & 0.5008 \\
CPS (mean) (SD) & $8.0(2.0)$ & $7.7(1.9)$ & 0.1950 \\
Hepatic encephalopathy (\%) & $\mathbf{9 3 ( 3 4 . 8 )}$ & $\mathbf{1 9 9 ( 2 3 . 4 )}$ & $\mathbf{0 . 0 0 0 3}$ \\
Ascites & $\mathbf{1 1 5 ( 4 3 . 1 )}$ & $\mathbf{2 9 2 ( 3 4 . 4 )}$ & $\mathbf{0 . 0 1 0 4}$ \\
Variceal bleeding & $19(7.1)$ & $50(5.9)$ & 0.4718 \\
Hepatocellular carcinoma & $9(3.4)$ & $17(2.0)$ & 0.2138 \\
Splenomegaly & $\mathbf{4 6 ( 1 7 . 2 )}$ & $\mathbf{8 6 ( 1 0 . 1 )}$ & $\mathbf{0 . 0 0 2 5}$ \\
& & &
\end{tabular}

MELD, model for end-stage liver disease; NAFLD, nonalcoholic fatty liver disease; CPS, Child-Pugh Score. * Patient characteristics were ascertained from the medical record at or prior to enrollment.
Table 5. Odds of opioid use among patient with NAFLD cirrhosis by patient characteristics*

\begin{tabular}{lll}
\hline & OR & $95 \%$ CI \\
\hline Age $\geq 62$ years (vs. $<62)$ & $\mathbf{0 . 7 0}$ & $\mathbf{0 . 5 1 - 0 . 9 6}$ \\
BMI $\geq 34 \mathrm{~kg} / \mathrm{m}^{2}$ (vs. $\left.<34\right)$ & 1.24 & $0.90-1.71$ \\
Depression & $\mathbf{2 . 2 9}$ & $\mathbf{1 . 6 6 - 3 . 1 8}$ \\
Osteoarthritis & $\mathbf{2 . 3 0}$ & $\mathbf{1 . 5 3}-\mathbf{3 . 4 6}$ \\
Rheumatologic diagnosis & $\mathbf{2 . 5 3}$ & $\mathbf{1 . 4 8}-\mathbf{4 . 3 0}$ \\
Ascites & 1.26 & $0.89-1.78$ \\
Hepatic encephalopathy & 1.39 & $0.97-2.00$ \\
\hline
\end{tabular}

Variables included in the stepwise model: age (years), sex, BMI, osteoarthritis, rheumatologic diagnosis, depression; MELD, ascites; HCC, and hepatic encephalopathy; significance level to remain in the model $=0.1{ }^{*}$ Patient characteristics were ascertained from the medical record at or prior to enrollment. NAFLD, nonalcoholic fatty liver disease; MELD, model for end-stage liver disease.
Fig. 1. Variable importance predictive of opioid use among patients with NAFLD using boosted trees. Gini improvement measure of relative importance estimates the difference in the prevalence of the outcome in the child nodes after the classification split [27]. AROC learning: 0.7602; AROC $_{\text {validation }}$ : 0.7321. Misclassification rate $_{\text {learning: }}$ 0.1796; misclassification rate $_{\text {validation }}: 0.1797$. Any opioid use at enrollment or 1 year prior: 100 layers. Multiple splits per tree: 3 . Sampling rate: 0.60 for rows and 0.40 columns. AUDIT, Alcohol Use Disorders Identification Test; GERD, gastroesophageal reflux disease. * Medical history on or prior to enrollment; ${ }^{* *}$ medical history of or drug use on or prior to enrollment.

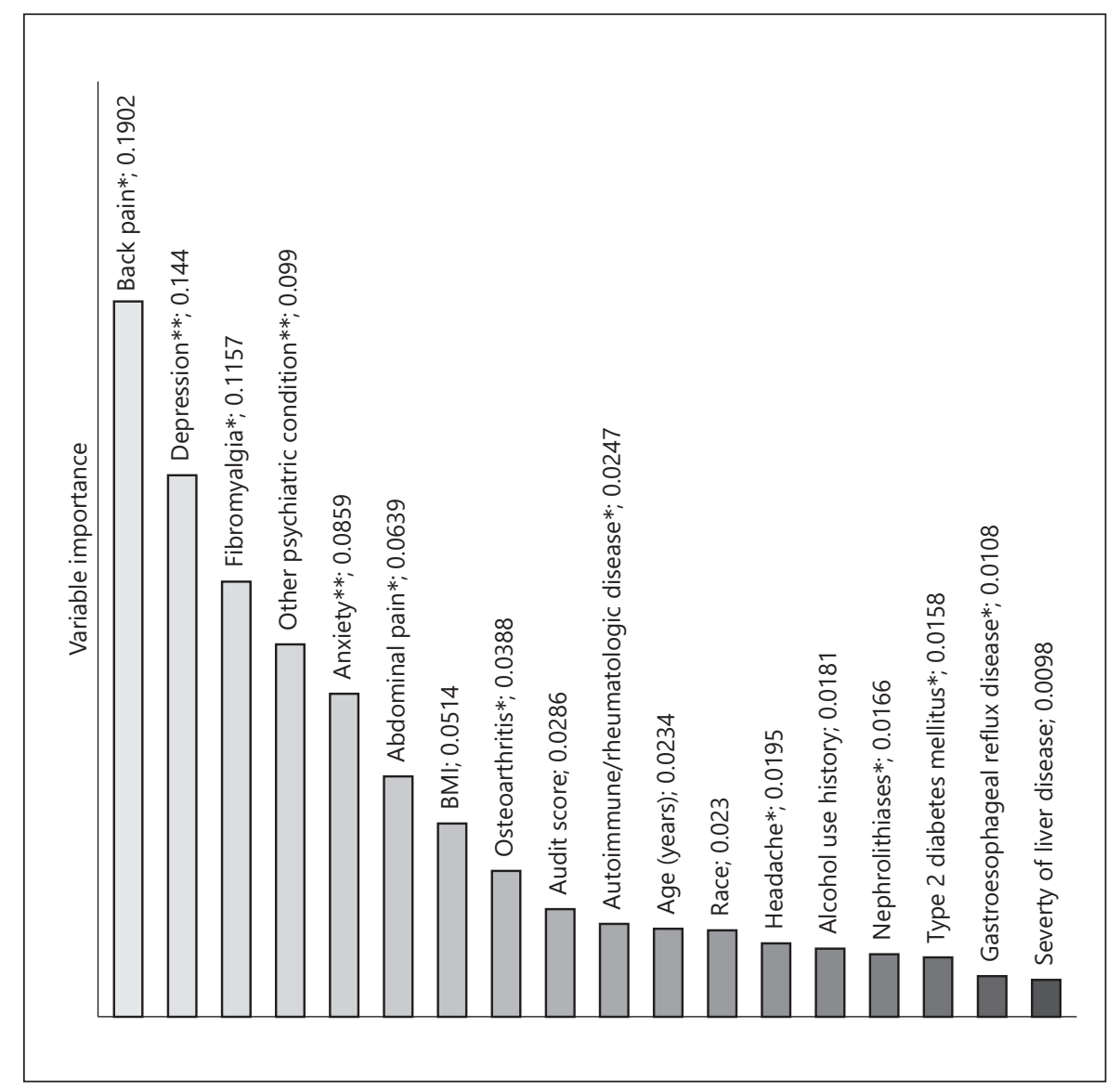


Table 6. CART estimating the predicted probability of opioid use among patients with NAFL*

Patient phenotypes

Probability of opioid use

Patients with depression

Depression, back pain

0.4578

Depression, no back pain, fibromyalgia

0.4368

Depression, no back pain, no fibromyalgia

0.2348

Patients without depression

No depression, abdominal pain

0.2142

No depression, no abdominal pain, back pain

0.2518

No depression, no abdominal pain, no back pain, anxiety

0.2067

No depression, no abdominal pain, no back pain, no anxiety, white or African American race $\quad 0.0710$

No depression, no abdominal pain, no back pain, no anxiety, other race

0.0210

CART, Classification and Regression Tree; AUDIT, Alcohol Use Disorders Identification Test; GERD, gastroesophageal reflux disease. ${ }^{*}$ Variable included in the model: age, sex, race, ethnicity, BMI, severity of liver disease, recent alcohol use, AUDIT score, history of diabetes mellitus, depression, anxiety, osteoarthritis, rheumatologic diagnosis, dyslipidemia, GERD, fibromyalgia, nephrolithiasis, headache, back pain, abdominal pain, ESRD, bariatric surgery, and any use of anxiolytics, antipsychotics, and antidepressants. AUROC, test: 0.7257, AUROC, validation: 0.7172, Misclassification Rate test: 0.1796, Misclassification Rate test: 0.1797. Depression: medical history or medication use for depression. Anxiety: medical history or medication use for anxiety.

\section{Discussion}

This cross-sectional study estimates real-world opioid use among patients with NAFLD. Nearly one-fifth of those with NAFLD and one-quarter of patients with NAFLD cirrhosis reported recent or ongoing opioid use. Opioid use was twice as common among those with NAFLD cirrhosis compared with NAFL and was associated with higher BMI, painful comorbidities, and psychiatric disease.

In the CART analysis, which allows for the identification of distinct subgroups of patients with NALFD who are likely to use opioids, both depression and back pain were associated with opioid use across patient subgroups. Approximately half of patients who had both a history of depression as well as a history of fibromyalgia were opioid users. The probability of opioid use among patients with back pain and depression was twice that of patients with back pain who were not depressed.

The results of both analyses, CART and logistic regression, suggested that depression and back pain were significantly associated with opioid use in patients with NAFLD. A logistic regression analysis estimated the average effect for the association between independent variables and the odds of an outcome, while a CART analysis can define clinical subgroups of patient's that are associ- ated with an increased probability of opioid use. The results of this classification analysis suggest that patients with NAFLD who also suffer from back pain, fibromyalgia, and/or depression might be at particularly high risk of future opioid use and may benefit from proactive alternative therapies to address pain, such as scheduled lowdose acetaminophen or referral to physical therapy and/ or a psychologist where appropriate.

Among patients with cirrhosis, the proportion of patients who were users of opioids in our cohort is slightly lower than prior studies reporting a $25-47 \%$ prevalence of opioid use among patients with cirrhosis $[8,9,13,29]$. This may be due to differences in how opioid use was ascertained or a reflection of our "real world" population of patients with NAFLD who were receiving care from both academic and community practices. These clinical practices may have less severe liver disease and fewer comorbidities than cohorts drawn exclusively from tertiary referral centers. In addition, given that our cohort was enrolled recently, this may be a reflection of the national trend toward reduced opioid prescribing [30].

This work supports previously findings in the literature which have suggested that opioid use was more common as liver disease progressed, with the highest reported prevalence of use in patients with NAFLD cirrhosis [8]. This could reflect an appropriate avoidance of NSAIDS 
given their risk of precipitating acute kidney injury and gastrointestinal bleeding or misinformation about the harms of acetaminophen use in the setting of cirrhosis. However, the higher reported use of non-opioid analgesics, including acetaminophen and NSAIDs, among patients with NASH and cirrhosis compared to NAFL suggests that this observation may be due to an increased prevalence of pain in those with higher BMI or lower levels of physical activity, both of which promote liver disease progression.

Another potential explanation for opioid use among patients with NAFLD is the association between metabolic syndrome and painful complications such as osteoarthritis due to increased mechanical load or diabetic neuropathy. Even after adjusting for BMI, osteoarthritis was significantly associated with opioid use. A lower pain threshold may also be attributable to higher levels of inflammatory cytokines seen in NAFLD $[9,31]$. It is possible that mood disturbances, seen more frequently in cirrhosis, and sleep disturbances, a common manifestation of hepatic encephalopathy, could contribute to opioid use even in the absence of pain [32]. In our cohort, fibromyalgia, anxiety, and depression were all associated with opioid use and hepatic encephalopathy was more commonly reported in opioid users.

Opioid use in patients with NAFLD is concerning given the potential role of opioids in sedentary behavior. Large prospective studies have demonstrated that the development of obesity is associated with a higher risk of chronic low back, neck, and shoulder pain and that this effect may be attenuated by exercise [33]. In our cohort, opioids were associated with obesity, suggesting that opioid use may predispose to the development or worsening of NAFLD.

While there are situations in which opioid prescription may be appropriate, particularly for acute pain, there are many reasons why opioids should be used cautiously in patients with NAFLD and cirrhosis [15, 34]. First, opioids have limited efficacy data for the treatment of chronic noncancer pain ( $>3$ months) $[34,35]$. Additionally, the use of long-acting opioids for noncancer pain is associated with increased risk for opioid abuse, opioid dependence, opioid overdose, and all-cause mortality compared to no opioid treatments or non-opioid analgesics $[34,36]$. Last, opioids have specifically been associated with poor outcomes in patients with cirrhosis including an increase in hepatic encephalopathy, length of stay, and hospital readmission [14, 37, 38].

Given opioids' limited long-term efficacy data and substantial risks, alternative options for patients with
NAFLD and pain should be fully explored [35]. Nonpharmacologic options should be considered first-line options in patients with NAFLD [3]. Exercise, which remains a cornerstone of treatment for NAFLD, has the dual benefits of improving hepatic steatosis in patients with NAFLD and decreasing back pain, joint pain, and symptoms of fibromyalgia [17-19,39-42]. This may be facilitated through early referral to physical therapy which is associated with reduced future opioid use [22]. Other nonpharmacologic treatment options that deserve additional study in patients with NAFLD and cirrhosis include multidisciplinary rehabilitation, mindfulnessbased stress reduction, and cognitive behavioral therapy [34]. Last, treatment for coexisting depression, anxiety, substance use disorders, and sleep disturbances may improve pain and reduce inappropriate opioid use. Acetaminophen ( $\leq 2 \mathrm{~g} /$ day) should be considered a first-line non-opioid analgesic option in cirrhosis given its safety and efficacy data [43]. Further study is needed to determine alternative analgesics or analgesic combinations that optimize physical function and limit adverse effects in patients with NAFLD and cirrhosis.

Our study has several strengths. It includes a large, real-world cohort of patients with NAFLD and, to our knowledge, represents the largest study to date examining use of opioids in this population. Many prior observational studies of patients with NAFLD have been limited to tertiary centers or large administrative databases, which may be more prone to referral bias, misclassification bias, and limited generalizability [44, 45]. In contrast, our cohort encompasses patients with NAFLD diagnosed by histology and clinical data, rather than billing codes, and includes patients from both community and academic settings. Therefore, our estimates of opioid use are likely to more accurately reflect patients with NAFLD receiving care in usual clinical practice.

There are a few limitations of this work. The crosssectional design of this study does not allow one to determine the temporal relationship between patient characteristics and current opioid use. Prospective studies, including the TARGET-NASH cohort, will help to shed light on the use of opioids in patients with NAFLD and the potential for associated complications. Moreover, the definition of opioid use is based on reports of a prescription for opioids at or within a year of enrollment and not on pharmacy fills. It is possible that patients who were prescribed but never took opioids were misclassified as opioid users and those who took nonmedical prescription opioids were misclassified as nonusers. Our cohort included primarily White and non-Hispanic patients who 
were actively receiving care in largely gastroenterology and hepatology practices across the United States. Patterns of opioid use may differ among other racial/ethnic groups or patients receiving care in other specialty or primary care clinics. Last, we were unable to differentiate between short-term and chronic opioid use.

Opioid use occurred in 1 of 5 patients with NAFLD and was more common among those who have more severe liver disease, anxiety, or depression. Given the safety concerns associated with opioid treatment, use of nonpharmacologic options and non-opioid analgesics should be considered for the treatment of pain in patients with NAFLD.

\section{Acknowledgements}

TARGET-NASH is sponsored by TARGET PharmaSolutions Inc. We would like to thank the study staff, nurses, health care providers, and participants for their contribution to this study. This is a collaboration of academic and community investigators, pharmaceutical partners, and NASH patient community advocates.

\section{Statement of Ethics}

This study was approved by WCG (WIRB Copernicus Group); reference number: 20161381. Clinical information from the electronic medical records, including patient narratives, laboratory results, pathology reports, and imaging data are extracted and uploaded into a secured database at 6-month intervals from the date of enrollment. Approvals from central and/or local institutional review boards are obtained prior to subject recruitment and at enrollment.

\section{Conflict of Interest Statement}

A.M. has no conflicts related to this work. S.W. is an employee of TARGET PharmaSolutions. A.S.L. has received research grants from BMS, Gilead, and TARGET PharmaSolutions (to University of Michigan) and is an advisor for TARGET PharmaSolutions. R.F.M. has no conflicts related to this work. H.N.T. has no conflicts related to this work. J.K. has no conflicts related to this work. C.S. is an employee of TARGET PharmaSolutions. B.A.N.-T. has no conflicts related to this work. A.S.B. is a consultant for TARGET PharmaSolutions.

\section{Funding Sources}

This research was supported in part by grants from the National Institutes of Health, T32 DK007634.

\section{Author Contributions}

All authors approved the final version of this manuscript. A. Sidney Barritt is the guarantor of this paper. Andrew M. Moon: Study concept and design, interpretation of data, drafting and critical revision of manuscript. Stephanie E. Watkins: Study concept, analysis, interpretation of data, drafting and critical revision of manuscript. Anna S. Lok: Study concept and design, interpretation of data, and critical revision of manuscript. Roberto Firpi-Morell: Study concept and design, interpretation of data, and critical revision of manuscript. Huy Ngoc Trinh: Study concept and design, interpretation of data, and critical revision of manuscript. Justin T. Kupec: Study concept and design, interpretation of data, and critical revision of manuscript. Cheryl Schoen: Data extraction, data analysis, interpretation of the data, and critical revision of the manuscript. Brent A. Neuschwander-Tetri: Study concept and design, interpretation of data, and critical revision of manuscript. A. Sidney Barritt: Study concept and design, interpretation of data, and critical revision of manuscript.

\section{References}

1 Dahlhamer J, Lucas J, Zelaya C, Nahin R, Mackey S, DeBar L, et al. Prevalence of chronic pain and high-impact chronic pain among adults: United States, 2016. MMWR Morb Mortal Wkly Rep. 2018;67(36):1001-6.

2 Scholl L, Seth P, Kariisa M, Wilson N, Baldwin G. Drug and opioid-involved overdose deaths: United States, 2013-2017. MMWR Morb Mortal Wkly Rep. 2018;67(5152): 1419-27.

3 Dowell D, Haegerich TM, Chou R. CDC guideline for prescribing opioids for chronic pain: United States, 2016. JAMA. 2016; 315(15):1624-45.

4 Perumpail BJ, Khan MA, Yoo ER, Cholankeril G, Kim D, Ahmed A. Clinical epidemiology and disease burden of nonalcoholic fatty liver disease. World J Gastroenterol. 2017;23(47): 8263-76.
5 Stone AA, Broderick JE. Obesity and pain are associated in the United States. Obesity. 2012; 20(7):1491-5.

6 Smuck M, Kao MC, Brar N, Martinez-Ith A, Choi J, Tomkins-Lane CC. Does physical activity influence the relationship between low back pain and obesity? Spine J. 2014;14(2): 209-16.

7 Hitt HC, McMillen RC, Thornton-Neaves T, Koch K, Cosby AG. Comorbidity of obesity and pain in a general population: results from the Southern Pain Prevalence Study. J Pain. 2007;8(5):430-6.

8 Rogal SS, Winger D, Bielefeldt K, Szigethy E. Pain and opioid use in chronic liver disease. Dig Dis Sci. 2013;58(10):2976-85.
9 Rogal SS, Bielefeldt K, Wasan AD, Lotrich FE Zickmund S, Szigethy E, et al. Inflammation, psychiatric symptoms, and opioid use are associated with pain and disability in patients with cirrhosis. Clin Gastroenterol Hepatol. 2015;13(5):1009-16.

10 Gutteling JJ, de Man RA, van der Plas SM, Schalm SW, Busschbach JJ, Darlington AS. Determinants of quality of life in chronic liver patients. Aliment Pharmacol Ther. 2006; 23(11):1629-35.

11 Rakoski MO, McCammon RJ, Piette JD, Iwashyna TJ, Marrero JA, Lok AS, et al. Burden of cirrhosis on older Americans and their families: analysis of the health and retirement study. Hepatology. 2012;55:184-91. 
12 Rakoski M, Goyal P, Spencer-Safier M, Weissman J, Mohr G, Volk M. Pain management in patients with cirrhosis. Clin Liver Dis. 2018; 11(6):135-40.

13 Rogal SS, Beste LA, Youk A, Fine MJ, Ketterer B, Zhang H, et al. Characteristics of opioid prescriptions to veterans with cirrhosis. Clin Gastroenterol Hepatol. 2019;17(6):1165-73. e3.

14 Acharya C, Betrapally NS, Gillevet PM, Sterling RK, Akbarali $\mathrm{H}$, White MB, et al. Chronic opioid use is associated with altered gut microbiota and predicts readmissions in patients with cirrhosis. Aliment Pharmacol Ther. 2017;45(2):319-31.

15 Dwyer JP, Jayasekera C, Nicoll A. Analgesia for the cirrhotic patient: a literature review and recommendations. J Gastroenterol Hepatol. 2014;29(7):1356-60.

16 Tapper EB, Baki J, Parikh ND, Lok AS. Frailty, psychoactive medications, and cognitive dysfunction are associated with poor patient-reported outcomes in cirrhosis. Hepatology. 2019;69(4):1676-85.

17 Busch AJ, Barber KA, Overend TJ, Peloso PM Schachter CL. Exercise for treating fibromyalgia syndrome. Cochrane Database Syst Rev. 2007; (4):CD003786.

18 Fransen M, McConnell S, Hernandez-Molina G, Reichenbach S. Exercise for osteoarthritis of the hip. Cochrane Database Syst Rev. 2014; (4):CD007912.

19 Fransen M, McConnell S, Harmer AR, Van der Esch M, Simic M, Bennell KL. Exercise for osteoarthritis of the knee. Cochrane Database Syst Rev. 2015;1:CD004376.

20 Hayden JA, van Tulder MW, Malmivaara A, Koes BW. Exercise therapy for treatment of non-specific low back pain. Cochrane Database Syst Rev. 2005;(3):CD000335.

21 Smart NA, King N, McFarlane JR, Graham PL, Dieberg G. Effect of exercise training on liver function in adults who are overweight or exhibit fatty liver disease: a systematic review and meta-analysis. Br J Sports Med. 2018 52(13):834-43.

22 Sun E, Moshfegh J, Rishel CA, Cook CE, Goode AP, George SZ. Association of early physical therapy with long-term opioid use among opioid-naive patients with musculoskeletal pain. JAMA Netw Open. 2018;1(8): e185909.
23 Barritt AS, Gitlin N, Klein S, Lok AS, Loomba $\mathrm{R}$, Malahias L, et al. Design and rationale for a real-world observational cohort of patients with nonalcoholic fatty liver disease: the TARGET-NASH study. Contemp Clin Trials. 2017;61:33-8.

24 United States Drug Enforcement Administration. Drug Scheduling. Available from: https: //www.dea.gov/drug-scheduling. Accessed 2020 Jun 28.

25 Wiesner R, Edwards E, Freeman R, Harper A, Kim R, Kamath P, et al. Model for end-stage liver disease (MELD) and allocation of donor livers. Gastroenterology. 2003;124(1):91-6.

26 Pugh RN, Murray-Lyon IM, Dawson JL, Pietroni MC, Williams R. Transection of the oesophagus for bleeding oesophageal varices. $\mathrm{Br}$ J Surg. 1973;60(8):646-9.

27 Lemon SC, Roy J, Clark MA, Friedmann PD, Rakowski W. Classification and regression tree analysis in public health: methodological review and comparison with logistic regression. Ann Behav Med. 2003;26(3):172-81.

28 Strobl C, Malley J, Tutz G. An introduction to recursive partitioning: rationale, application, and characteristics of classification and regression trees, bagging, and random forests. Psychol Methods. 2009;14(4):323-48.

29 Konerman MA, Rogers M, Kenney B, Singal AG, Tapper E, Sharma P, et al. Opioid and benzodiazepine prescription among patients with cirrhosis compared to other forms of chronic disease. BMJ Open Gastroenterol. 2019;6(1):e000271.

30 Guy GP Jr, Zhang K, Bohm MK, Losby J, Lewis $B$, Young R, et al. Vital signs: changes in opioid prescribing in the United States, 20062015. MMWR Morb Mortal Wkly Rep. 2017; 66:697-704.

31 Kugelmas M, Hill DB, Vivian B, Marsano L, McClain CJ. Cytokines and NASH: a pilot study of the effects of lifestyle modification and vitamin E. Hepatology. 2003;38:413-9.

32 Schuh-Hofer S, Wodarski R, Pfau DB, Caspani O, Magerl W, Kennedy JD, et al. One night of total sleep deprivation promotes a state of generalized hyperalgesia: a surrogate pain model to study the relationship of insomnia and pain. Pain. 2013;154(9):1613-21.

33 Nilsen TI, Holtermann A, Mork PJ. Physical exercise, body mass index, and risk of chronic pain in the low back and neck/shoulders: longitudinal data from the Nord-Trondelag Health Study. Am J Epidemiol. 2011;174(3): 267-73.
34 Centers for Disease Control and Prevention. CDC guideline for prescribing opioids for chronic pain: United States, 2016. MMWR Recomm Rep. 2016 Mar 18;65(1):1-49.

35 Busse JW, Wang L, Kamaleldin M, Craigie S, Riva JJ, Montoya L, et al. Opioids for chronic noncancer pain: a systematic review and meta-analysis. JAMA. 2018;320(23):2448-60.

36 Ray WA, Chung CP, Murray KT, Hall K, Stein CM. Prescription of long-acting opioids and mortality in patients with chronic noncancer pain. JAMA. 2016;315(22):2415-23.

37 Moon AM, Jiang Y, Rogal SS, Becker J, Barritt AS. In inpatients with cirrhosis opioid use is common and associated with length of stay and persistent use post-discharge. PLoS One. 2020;15(2):e0229497.

38 Moon AM, Jiang Y, Rogal SS, Tapper EB, Lieber SR, Barritt AS. Opioid prescriptions are associated with hepatic encephalopathy in a national cohort of patients with compensated cirrhosis. Aliment Pharmacol Ther. 2020 Mar. 51(6):652-60.

39 Romero-Gomez M, Zelber-Sagi S, Trenell M. Treatment of NAFLD with diet, physical activity and exercise. J Hepatol. 2017;67:829-46.

40 St George A, Bauman A, Johnston A, Farrell $\mathrm{G}$, Chey T, George J. Independent effects of physical activity in patients with nonalcoholic fatty liver disease. Hepatology. 2009;50(1): 68-76.

41 Senna MK, Sallam RA, Ashour HS, Elarman M. Effect of weight reduction on the quality of life in obese patients with fibromyalgia syndrome: a randomized controlled trial. Clin Rheumatol. 2012;31(11):1591-7.

42 Mork PJ, Vasseljen O, Nilsen TI. Association between physical exercise, body mass index, and risk of fibromyalgia: longitudinal data from the Norwegian Nord-Trondelag Health Study. Arthritis Care Res. 2010;62:611-7.

43 Benson GD. Acetaminophen in chronic liver disease. Clin Pharmacol Ther. 1983;33(1):95101.

44 Lazo M, Hernaez R, Eberhardt MS, Bonekamp S, Kamel I, Guallar E, et al. Prevalence of nonalcoholic fatty liver disease in the United States: the Third National Health and Nutrition Examination Survey, 1988-1994. Am J Epidemiol. 2013;178:38-45.

45 Adams LA, Lymp JF, St Sauver J, Sanderson SO, Lindor KD, Feldstein A, et al. The natural history of nonalcoholic fatty liver disease: a population-based cohort study. Gastroenterology. 2005;129(1):113-21. 